\title{
A LIST OF COCCIDAE AFFEC'TING VARIOUS GENERA OF PLANTS.
}

\author{
By E. ERNEST GREEN, F.E.S., F.Z.S. \\ (Continued from page 89)
}

DACRYDIUM (Coniferae).

Ctenochiton dacrydii.

DACTYLIS (Gramineae).

Pseudococcus walkeri.

Ceroputo volynicus.

Luzulaspis luzulae.

DAHLIA (Compositae).

Pseudococcus affinis.

Pulvinaria psidii.

DAI.BERGIA (Leguminosae).

Monophlebus dalbergiae, octocaudata.

TACHARDIA lacca.

LeCANiUm expansum.

Aspidiotus trilobitiformis, lataniae, simillimus, transparens.

DALEA (Leguminosae). Ceroplastodes daleae.

DAMMARA (Coniferae).

Pseudococcus aurilanatus.

DANAE (Liliaceae).

Fiorinia pellucida.

DANTHONIA (Gramineae).

Eriococcus danthoniae.

DAPHNE (Thymeliaceae).

RHzzococcus gnidii.

Pseudococcus similans.

LECANIUM magnoliarum.

Aspidiotus caldesii, hederae, camel-

liae, lataniae.

DASYLIRION (Bromeliaceae).

LeCaniodiaspis yuecae.

Pseudococcus dasylirii.

DATURA (Solanaceae).

Pseudococcus virgatus.

Aspidiotus hederae.
DAVALLIA (Filices).

HeMICHIONASPIS aspidistrae.

LEPIDOSAPHES ocellata.

DAVIESIA (Leguminosae).

Pulvinaria tecta.

Asterolecanium stypheliae.

Chionaspis nitida.

Poliaspis nitens.

Aspidiotus hederae.

LEPIDOSAPHES casuarinae.

DEBREGAESIA (Urticaceae).

Pseudococcus debregaesiae.

Ceroplastes ceriferus.

Hemichionaspis mussaendae, minor.

DENDROBIUM (Orchidaceae).

LEUCASPIS stricta.

Aspidiotus dictyospermi.

LEPIDOSAPHES cocculi.

DENDROCALAMUS (Gramineae).

Asterolecanium bambusae, coronatum.

Antonina bambusae.

Chionaspis angusta.

DENDROPHTHORA (Loranthaceae).

Pulvinaria dendrophthorae.

DERRIS (Leguminosae).

LEPIDOSAPHES dilatilobis.

DESCHAMPSIA (Gramineae).

Eriopeltis festucae.

DESMANTHUS (Leguminosae).

Ceronema africana.

DEUTZIA (Philadelphaceae).

Aspidiotus perniciosus.

DEVERRA (Umbelliferae).

Chionaspis bilobis. 
DIANELLA (Liliaceae). LEUCASPIS cockerelli. Parlatoria proteus.

DICHAPETALUM (Chailletiaceae). Aspidiotus maeandrius.

DICHOPSIS (Sapotaceae). ICERYAE seychellarum. VINSONIA stellifera.

DICTYOSPERMA (Palmaceae). Pinnaspis buxi.

Aspidiotus dictyospermi, articulatus, ficus.

DIELYTRA (Fumariaceae). LECANIUM hesperidum.

DILLENIA (Dilleniaceae). LECANIUM cocophyllae.

DILLWYNIA (Leguminosae). LECANIUM pingue. Pulvinaria contexta. Poliaspis exocarpi.

DIOON (Cycadaceae). Poliaspis cycadis. Diaspis zamiae.

DIOSCOREA (Dioscoreaceae). Aspidiotus hartii, destructor, trans-

DIOSMA (Rutaceae). [parens, LECANIUM oleae. Aspidiotus camelliae.

DIOSPYROS (Ebenaceae). ICERYA seychellarum. LeCANIODIASPIS tessellata. Phenacoccus pergandei. Ceroplastes ciripediformis. Pulvinaria citricola. Lecanium corni, bicruciatum. DiAsPis pentagona. Aspidiotus perniciosus, dictyospermi, duplex, aurantii, orientalis. Parlatoria pergandei-phyllanthi.

DIPTEROCARPUS (Dipterocarpaceae). TACHARDIA lacea.

DIRCA (Thymeliaceae). Chionaspis lintneri.

DISPORUM (Liliaceae). Aspidiotus hederae.

DISTICHLIS (Gramineae). SpHaERococcus distichlium. ErIococcus salinus.

DISTYLIUM (Hamamelideae). Chionaspis latissima.
DOBERA (Salvadoraceae). DinasPIS reticulata.

DODONAEA (Sapindaceae). WaLkERIANA senex. Pulvinaria dodonaeae, thompsoni, psidii.

DOLICHOS (Leguminosae). Ceroplastodes cajani. INGLISIA bivalvata. LECANIUM longulum.

DOMBEYA (Sterculiaceae). ICERYA jacobsoni.

DOODIA (Filices). Alecanopsis filicum.

DORYCNIUM (Leguminosae). AsTEROLECANIUM fimbriatum.

DOVYALIS (Flacourtiaceae). Howarda biclavis. Cerococcus ornatus.

DRABA (Cruciferae). Asterolecanium fimbriatum.

DRACAENA (Liliaceae). LECANIUM hesperidum. Hemichionaspis dracaenae, aspidistrae.

Pinnaspis buxi. Chionaspis tangana. Aspidiotus lauretorum, tinerfensis, dictyospermi, lataniae, hederae.

LEUCASPIS cockerelli.

DRIMYS (Magnoliaceae). IngLisia patella. Psfudoparlatoria parlatorioides. LEPIDOSAPHES drimydis.

DRYANDRA (Proteaceae). Ceronema dryandrae. Aspidiotus dryandrae. Parlatoria dryandrae.

DRYMUS. Aspidiotus moreirae, pisai.

DRYPETES (Euphorbiaceae). LECANIUM hemisphaericum.

DURANTA (Verbenaceae). Pulvinaria psidii. LeCanium oleae. ORTHEZIA insignis.

DURIO (Malvaceae). CHIONASPIS durionis.

DYSOXYLON (Meliaceae). Chionaspis dysoxyli. 
DYSOXYLON (Meliaceae)—coni.

Aspidiotus dysoxyli.

LEPIDOSAPHES pyriformis.

EARINA (Orchidaceae). Ctenochiton elongatus.

ECHINOCACTUS (Cactaceae).

Diaspis echinocacti.

ECHITES (Apocynaceae).

Phenacoccus mangiferae.

Aspidiotus trilobitiformis.

EDGEWORTHIA (Thymeliaceae).

Pseudococcus edgeworthiae.

EHRETIA (Boragineae).

Aspidiotus replicatus.

ELAEAGNUS (Elaeagnaceae).

LECANIUM bicruciatum, persicae, hesperidum, nigrofasciatum.

Ceroplastes floridensis.

Chionaspis difficilis, elaeagni, vitis.

DIASPIS erawii.

Aspidiotus camelliae, britannicus, rossi.

AONIDIA elaeagni.

LEPIDOSAPHES citricola, crawii, ulmi.

ELAEIS (Palmaceae).

Ceroplastes actiniformis.

HeMICHIONASPIS marchali.

Aspidiotus elaeidis.

IsCHNASPIS filiformis.

ELAEOCARPUS (Tiliaceae).

ERIococcus pallidus.

Pseudococcus scrobicularum.

LECANIUM formicarii.

ERIOCHITON spinosus.

Ctenochiton elaeocarpi, flavus.

InGLISIA ornata.

HeMichionaspis scrobicularum.

ELAEODENDRON (Celastraceae).

Fiorinia elaeodendri, plana.

ELAPHRIUM (Burseraceae).

Ceroplastes ceriferus.

ELLIPANTHUS (Connaraceae). Chionaspis vitis.

ELYMUS (Gramineae). RIPERSIA smithii.

ELYTROPAPPUS (Compositae). SPHAERococcus africanus. Pseudococcus elizabethae.
EPACRIS (Epacridaceae).

ERIococcus multispinosus-laevigatus.

LECANIODIASPIS microcribraria.

EPHEDRA (Gnetaceae).

Pseudococcus ephedrae.

Lichtensia ephedrae.

Philephedra ephedrae.

Stotzia striata.

Finippia ephedrae.

Dinaspis ichesii.

LeUCaspis ephedrae.

Aspidiotus trabuti, ephedrarum.

Parlatoria ephedrae.

EPIDENDRUM (Orchidaceae).

Asterolecanium epidendri.

EPIGAEA (Ericaceae).

Aspidiotus epigaeae.

ERANTHEMUM (Acanthaceae).

ICERYA seychellarum, sulfurea.

LECANIUM hemisphaericum, depressum.

Pulvinaria jacksoni.

EREMOPHILA (Myoporaceae).

Aspidiotus coralinus, hederae.

ERICA (Ericaceae).

ORthezIa maenariensis, urticae.

AsteroleCanium fimbriatum.

Eriococcus devoniensis, ericae.

RIPERsia halophila.

Pseudococcus ericicola.

Pulvinaria ericae.

LeCANIUM oleae, hemisphaericum.

DIASPIS ericicola.

Chionaspis salicis.

AdIscodiaspis ericicola.

Aspidiotus hederae, bavaricus.

LEPIDOSAPHES ulmi.

ERIGERON (Compositae).

Pseudococcus neomexicanus.

Ceroplastes cultus, cuneatus.

ERIOBOTRYA (Rosaceae).

ICERYA purchasi.

Ceroplastes vinsonii.

DIASPIS pentagona.

Aspidiotus dictyospermi, aurantii.

Parlatoria oleae, pergandei.

ERIODENDRON (Malvaceae), 'Silk Cotton.'

Aspidoproctus giganteus.

LECANIUM intimum, nigrum. 
ERIODENDRON (Malvaceae)-cont. AsPIDIOTUs pedroniformis.

ERIODICTYON (Hydrophyllaceae). Pseudococcus yerba-santae.

ERIOGONUM (Polygonaceae).

ERIococcus palmeri, eriogoni.

Pseudococcus maritimus.

ERIUM eriogoni.

ERYTHEA (Palmaceae).

Comstockiella sabalis.

HEMICHIONASPIS aspidistrae.

ERYTHRASPIS

Aspidiotus camelliae, erythraspidis.

ERYTHRINA (Leguminosae).

Pseudococcus crotonis.

LeCanium tenebricophilum, oleae.

Pulvinaria maxima.

Eriochiton theae.

Diaspis pentagona.

Aspidiotus dictyospermi, pustulans.

LEPIDOSAPHES erythrinae.

ERYTHROPALUM (Olaeaceae).

LECANIUM cocculi.

EUCALYPTUS (Myrtaceae).

MoNOPHLEBUS fortis, crawfordi, crawfordi-levis, crawfordi-pilosior.

Gueriniella serratulae.

Callipappus australis, immanis.

Aргомоврна attenuata, bauerleni, calycina, conica, duplex, minor, ellipsoides, floralis, helmsii, karschi, maliformis, munita, munitatricornis, ovicola, ovicola-glabra, ovicoloides, pedunculata, pharetrata, pileata, pomiformis, rosaeformis, rugosa, sessilis, sloanei, strombylosa, thorntoni, thorntoninux, umbellata, urnalis, variabilis, urnalis-schraderi, cucurbita, dipsaciformis, excupula, fletcheri.

OpISThoscelis conica, fibularis, globosa, maculata, mammularis, maskelli, nigra, pisiformis, serrata, spinosa, subrotunda, verrucula.

Ascelis attenuata, echiniformis, praemollis, schraderi.

WARBURTONIA frenchi.

LeCaniodiaspis eucalypti, frenchi, convexus, newmani.

GossyPaRIA confluens.
Eriococcus confusus, coriaceus, eucalypti, simplex, simplex-dealbata, tepperi, tricarinatus, serratilobis, crofti, apiomorphae, gregarius, irregularis, picta, tessellatus.

SpHaERococcus elevans, obscuratus, pustulans.

SpHaERococopsis inflatipes.

Ourococcus cobbii, eucalypti.

LACHNODIUS eucalypti, lectularius.

Pseudococcus lobulatus.

TAChardia melaleucae.

LECANIUM oleae.

Ctenochiton eucalypti.

Ceronema caudata.

Pulvinaria maskelli.

Chionaspis assimilis, ethelae, eugeniae, formosa, eucalypti, frenchi, angusta.

Aspidiotus extensus, perniciosus, camelliae, subrubescens, rossi, acaciae, eucalypti, alatus, confusus, rubribullata, subrubescenscorticoides, rossi-victoriae, fuscus, tasmaniae, miniatae, ficus, amplius, cyanophylli, articulatus, personatus.

Maskelita globosa.

LEPIDOSAPHES cordylinidis, grisea, lidgetti, bicornis, eucalypti, bicornis-alba.

Fiorinia lidgetti.

EUCHARIS (Amaryllidaceae).

AsterolecaniUm aureum.

HeMichionaspis minor.

EUGENIA (Myrtaceae).

Llanveia primitiva-pimentae.

Capulinia jaboticabae, crateriformans.

ERIOIDES cuneiformis.

CARPochloroides viridis.

StictoleCaniUm ornatum.

LeCaniUm tessellatum, jaboticabae, bicruciatum, psidii, mangiferae.

Pulvinaria eugeniae, psidii, grabhami.

Ceroplastes fairmairii, formosus, rubens.

VINSONIA stellifera. 


\section{List of Coccidae affecting various Genera of Plants}

EUGENIA (Myrtaceae) - cont.

Edwallia rugosa.

Chionaspis eugeniae.

Fiorinia fioriniae.

Aspidiotus fimbriatus, virescens, hederae, ficus, camelliae, destructor, personatus.

ODONaSPIS pimentae.

LEPIDOSAPHES rubrovittatus, ungulata.

Parlatoria oleae, proteus.

EUONYMUS (Celastraceae).

LeCANIUM oleae, coryli, nigrofasciatum, capreae.

Pulvinaria camelicola, euonymi, innumerabilis, betulae, floceifera.

Diaspis pentagona.

Chionaspis eitri, euonymi, acuminata, salicis.

Aspidiotus euonymi, perniciosus, aurantii, rossi, camelliae, dictyospermi, ficus, lataniae.

Parlatoria theae-euonymi.

EUPATORIUM (Compositae).

ORTHEZIA insignis.

AsteroleCANIUM fimbriatum.

EUPHORBIA (Euphorbiaceae).

Palaeococcus rosae.

ICERYA euphorbiae.

WALKERIANA euphorbiae.

Orthezia urticae.

Asterolecanium fimbriatum.

Pseudococcus virgatus, longispinus.

Lecanium longulum.

Ceroplastes euphorbiae.

Cerococcus alluaudi.

DIASPIS conservans, barrancorum, antiquorum.

Aspidiotus trilobitiformis-darutyi, ferox, magnus, euphorbiae, fissus, taorensis, lenticularis, biformis, hederae.

Cryptaspidiotus austroafricanus.

Lepidosaphes ulmi.

Parlatoria mangiferae.

EUPHRASIA (Scrophulariaceae).

ASTEROLECANIUM fimbriatum.

EURYA (Ternstroemiaceae).

LECANIUM ochnaceae.

Pulvinaria aurantii, psidii.
EURYA (Ternstroemiaceae)—cont. Aspidiotus duplex.

LEPIDOSAPHES euryae.

EURYCLES (Amaryllidaceae).

CHIONASPIS dilatata.

EUTERPE (Palmaceae).

Diaspis boisduvallii.

EVODIA (Rutaceae).

Chionaspis acuminata.

Dinaspis permutans.

EXCOECARIA (Euphorbiaceae).

LECANIUM longulum.

Ceroplastes excoecariae.

EXOCARPUS (Thymeliaceae).

Cerococcus bryoides, bryoidesstellatus.

Pseudococcus hibbertiae.

Ceroplastodes melaleucae.

Poliaspis exocarpi.

Aspidiotus junctlobius.

LEPIDOSAPHES casuarinae, subspicu-

[lifera.

FABIANA (Solanaceae).

Pulvinaria argentina.

Ceroplastes longiseta.

Aspidiotus fabianae.

FAGONIA (Zygophyllaceae).

Aspidiotus nigra, hederae.

FAGRAEA (Loganiaceae).

LeCANIUM psidii.

DiAsPIS fragraeae.

FAGUS (Corylaceae) 'Beech.'

Coelostomidia assimilis, pilosa.

Phenacoleachia zealandica.

Cerococcus fagi.

Fagisuga triloba.

Platypyga fagi.

Eriococcus fagicorticis, pallidus, raithbyi, aceris.

RHIzococcus intermedius, maculatus, pulchellus, totarae, cavellei.

Cryptococcus fagi.

Pseudococcus iceryoides, obtectus, aceris, newsteadi.

Ripersia fagi.

Pulvinaria innumerabilis, betulae.

INGLISIA fagi.

Aspidiotus an'ylus, forbesi, ostreiformis, perniciosus.

LEPIDOSAPHES ulmi. 
FATSIA (Araliaceae).

Pulvinaria psidii.

Protopulvinaria japonica.

FERONIA (Rutaceae).

Anomalococcús cremastogastri.

FESTUCA (Gramineae).

Eriococcus festucae.

Ripersia festucae.

Trionymus californicus.

ERIopeltis festucae, lichtensteinii.

FICUS (Moraceae).

Aspidoproctus verrucosus.

Drosicha maskelli, lichenoides.

MoNophlebus octocaudata.

ICERYA aegyptiaca, maxima, purchasi, seychellarum.

Gueriniella serratulae.

TaChardia lacca, fici, longisetosa.

Asterolecanium pustulans.

LECANIODIASPIS africana.

Anomalococcus cremastogastri.

ERIococcus crispus, lagerstroemiae.

Pseudococcus ficus, longispinus, setosus, adonidum, citri, crotonis, virgatus, virgatus-madagascariensis.

Phenacoccus mangiferae.

Strctococcus formicarii.

LeCanium ficus, longulum, depressum, nigrella, plebeium, nigrum, ficinum, oleae, persicae, tessellatum, crassum, hesperidum, desolatum, hemisphaericum, formicarii, expansum, expansum-javanicum, ramakrishnae, carpenteri.

HeMULECANIUM imbricans.

Pulvinaria ficus, psidii, psidiiphilippina, jacksoni, cupaniae.

Lichtensia lutea.

Ceroplastes floridensis, rubens, rusci, townsendi-percrassus, ficus, quadrilineatus, gowdeyi, coniformis, ceriferus, galeatus.

Diaspis gennadii.

Schizaspis lobata.

Chionaspis manni, fici.

HowaRdia biclavis.

Hemichionaspis aspidistrae, minor, fici, minima.

LeUCASPIS japonica.

Ann. Biol. IV
FICUS (Moraceae)-cont.

Aspidiotus cyanophylli, cydoniae, camelliae, trilobitiformis, articulatus, ficus, aurantii, africanus, sylvatica, fossor, obsita, orientalis, dictyospermi, personatus, lataniae.

AONIDIA planchonioides.

Gymnaspis ficus.

LEPIDOSAPHES citricola, ficus, ficifolii, mexicana, minima, pinniformis, conchiformis, luzonica.

ISCHNASPIS filiformis.

FILICES 'Ferns.'

Tessarobelus guerinii.

Orthezia cheilanthi.

ErIococcus insignis.

Macrocerococcus superbus.

Pseudococcus longispinus.

RIPERsia filicicola.

LeCANIUM tessellatum, hesperidumalienum, cockerelli, filicum, hemisphaericum, mori, diversipes, oleae, longulum.

Pulvinaria mammeae, floccifera.

Ceroplastes rubens.

Ctenochiton depressus.

Alecanopsis filicum.

Chionaspis dilatata, dubia.

Hemichionaspis aspidistrae.

Poliaspis media.

LEPIDOSAPHES phymatodidis, ocellata, desmidioides.

FILICIUM (Burseraceae).

TACHARDIA albizziae.

FLACOURTIA (Flacourtiaceae).

Pseudococcus longispinus.

DiAsPIS pentagona-flacourtiae.

Howardia biclavis.

Parlatoria cingala.

FOUQUIERIA (Tamaricaciae).

Lecaniodiaspis rufescens.

FRAGARIA (Rosaceae) 'Strawberry.'

ICERYA genistae.

ORthezIa insignis.

Diaspis rosae.

Aspidiotus perniciosus.

FRAXINUS (Oleaceae) 'Ash.'

Fonscolombia fraxini.

Phenacoccus aceris, pettiti. 


\section{List of Coccidae affecting various Genera of Plants}

FRAXINUS (Oleaceae) - cont.

LeCANIUM cerasifex, fraxini, oleae, nigrofasciatum, corni, robiniaesubsimile.

Pulvinaria fraxini, betulae.

Ericerus pela.

Diaspis pentagona.

Chionaspis salicis.

Aspidiotus ancylus, juglansregiaealbus, perniciosus, townsendi, vagabundus, pyri.

LePIDOSAPHeS ulmi.

PARLatoria oleae, affinis.

FRENELLA (Coniferae).

ErIUM frenellae.

FREYCINETIA (Pandanaceae).

Pseudococcus montanus.

FUCHSIA (Onagraceae).

Pseudococcus citri, longispinus.

Ceroplastes albolineatus.

Aspidiotus camelliae.

LEPIDOSAPHES lactea.

FUMARIA (Papaveraceae).

Macrocerococcus superbus.

Ceroputo superbus.

FUNTUMIA (Apocynaceae).

Pulvinaria psidii.

Ceroplástes ceriferus.

Chionaspis funtumiae, lutea.

FURCRAEA (Amaryllidaceae).

LECANIUM hesperidum, depressum.

Aspidiotus furcraeicola, hederae.

GAERTNERA (Loganiaceae).

Fiorinia scrobicularum.

GAHNIA (Cyperaceae).

LEPIDOSAPHES cordylinidis.

GALIUM (Rubiaceae).

Orthezia urticae.

Pseudococcus pulverarius.

Aspidiotus niger.

GARCINIA (Guttiferae).

LECANIUM zonatum.

Pulvinaria psidii.

Ceroplastes rubens.

VInsonia stellifera.

Aspidiotus greeni, dictyospermi, rossi, rossi-ferrandii.

Fiorinia frontecontracta.

Parlatoria proteus.
GARDENIA (Rubiaceae).

Drosicha maskelli.

Orthezia insignis.

LECANIUM viride, hemisphaericum, hesperidum, oleae.

Protopulvinaria longivalvata.

Ceroplastes ceriferus.

Howardia biclavis.

Aspidiotus articulatus, hederae, tayabanus.

GARRYA (Cornaceae).

Aspidiotus hederae.

GARUGA (Amyridaceae).

TACHARDIA lacca.

GAULTHERIA (Ericaceae).

HeMICHIONASPIS minor.

GAYLUSSACIA (Ericaceae).

Aspidiotus perniciosus.

GEIJERA (Rutaceae).

Frorinia geijerae.

Aspidiotus hederae, rossi.

GELONIUM (Euphorbiaceae).

INGLISIA chelonioides.

ChIONASPIS varicosa.

Fiorinia proboscidaria, saprosmaegelonii.

GELSEMIUM (Loganiaceae).

Aspidiotus hederae.

GENIOSTOMA (Loganaceae).

Ctenochiton elongatus.

GENISTA (Leguminosae).

ICERYA genistae.

Asterolecanium fimbriatum.

LECANIUM genistae.

DIASPIS pentagona.

Chionaspis salicis.

Aspidiotus hederae, niger.

Lepidosaphes ulmi.

GERANIUM (Geraniaceae).

Orthezia urticae, cataphracta.

Asterolecanium fimbriatum, pustulans.

Pseudococcus bechuanae.

Diaspis pentagona.

GIGANTOCHLOA (Gramineae). Asterolecanium bambusae.

Antonina bambusae.

Odonaspis canaliculata.

GLAUX (Primulaceae).

Orthezia urticae. 
GLEDITSCHIA (Leguminosae).

Pseudococcus burnerae.

LeCanium cynobati, rugosum, oleae.

Diaspis pentagona.

Chionaspis ortholobis, gleditsiae, spinicola.

Aspidiotus forbesi, fernaldi, africanus, ostreaeformis.

GLOBULARIA (Globulariaceae).

Asterolecanium rehi, fimbriatum.

CRYPTOPHYLlaspis bornmulleri.

Aspidiotus labiatarum.

GLYCINA (Leguminosae).

LECANIUM wistariae.

GLYCOSMIS (Rutaceae).

VINSONIA stellifera.

GNETUM (Gnetaceae).

Pseudoparlatoria cristata.

Cryptoparlatoria uberifera.

GOODENIA (Goodeniaceae).

ICERYA aegyptiaca.

GOSSYPIUM (Malvaceae) 'Cotton.'

Cerococcus hibisci.

Pseudococcus citri, virgatus, perniciosus, filamentosus, corymbatus, longispinus.

Phenacoccus gossypii.

LECANIUM nigrum.

Pulvinaria jacksoni, maxima.

INGLISIA malvacearum.

Hemichionaspis minor, townsendi, aspidistrae-gossypii.

GOURLIEA (Leguminosae).

Pseudococcus percerosus.

GRAPTOPHYLLUM (Acanthaceae).

Pseudococcus virgatus.

LECANIUM nigrum.

IsCHNASPIS filiformis.

'GRASSES' (Gramineae).

Monophlebus fulleri.

WALKERIANA africana.

ICERYA pilosa, purchasi.

Margarodes hamelii, newsteadi, peringueyi, ruber, papillosus, niger.

ORthezia cataphracta, graminis, martelli, urticae.

Newsteadia floceosa.

Macrocerococcus superbus.

ERIococcus graminis, greeni, insignis, kemptoni, quercus, nativus, ruber, tenuis, formicola, salinus, festucae, inermis.

Pseudococcus arecae, calceolariae, graminis, herbicola, hibernicus, neomexicanus-alkalinus, nubicola, poae, roseotinctus, salinus, segregatus, walkeri, calceolariae-minor, bantu, elongatus, natalensis, socialis, mallyi, caffra, flagrans, timberlakei, aridorum, citri, pulverarius, kandyensis.

Phenacoccus cholodkovskyi, graminicola, graminis.

Ceroputo calcitectus, volynicus, superbus.

Natalensia fulleri.

RIPERsia festucae, halophila, montana,porterae, salmonacea, subterranea, tenuipes, tomlini, trichura, corynephori, sporoboli, Jibera, oryzae, smithii, occulta, globata.

Trionymus perrisii, nanus, californicus, violaceus, insularis.

RIPERSiella kelloggi, maritima.

Geococcus radicum.

Mrcrococcus similis.

Antonina graminis, parrotti, purpurea, indica, phragmitis, natalensis, transvaalensis, maritima.

LECANIUM anthurii, tenuivalvatum.

LECANOPSIS brevicornis, longicornis, formicarum, butleri.

Luzulaspis luzulae.

Spermococcus fallax.

ExaERETopus caricis.

Eriopeltis festucae, lichtensteinii, brachypodii, coloradensis.

Parafatrmatria bipartita, gracilis.

ACLERDA californica, digitata, obscura, subterranea, signoreti.

Diaspis uncinati.

EPIDIASPIS subterranea.

Chionaspis herbae, natalensis, graminis, spartinae.

Немпсніоnaspis aspidistrae.

Aspidiotus bilobis, hederae, graminella, sacchari, marlatti, panici.

Odonaspis janeirensis, graminis, ruthae.

LEPIDOSAPHES ampelodesmae. 


\section{List of Coccidae affecting various Genera of Plants}

GREVILLEA (Proteaceae).

WALKERIANA cinerea.

Aspidoproctus maximus.

Asterol.eCanidu pustulans.

Pseudococcus grevilleae, filamentosus, citrophilus.

ErIUM globosum, newmani.

LECANIUM longulum, hemisphaericum-hibernaculorum.

HEMICHIONASPIS minor.

Howardia biclavis.

Aspidiotus hederae, camelliae.

LEPIDOSAPHES grevilleae.

GREWIA (Tiliaceae).

TACHaRdia lacca.

ERIUM newmani.

Tylococcus formicarii.

LeCANiUM oleae.

INGLISIA castilloae.

Chionaspis vitis.

Frorinia tumida, secreta.

Cryptophyllaspis occultus, occultus-elongatus.

GRINDELIA (Compositae).

Palaeococcus townsendi.

LECANIUM assimile.

GRISELINIA (Cornaceae).

LEUCASPIS gigas.

GRISLEA (Lythraceae).

Aspidiotus moorei.

GROSSULARIA (Grossulariaceae).

LeCanium armeniacum, coryli, eynobati, rehi, ribis, persicae.

Pulvinaria occidentalis.

Fiorinta grossulariae.

Aspidiotus forbesi, perniciosus.

GUAIACUM (Zygophyllaceae).

Palaeococcus rosae.

LeCANIUM tessellatum.

Ceroplastes floridensis.

Aspidiotus aurantii.

GUAZUMA (Sterculiaceae).

TACHARDIA rotundata.

ERIOcoccus aurescens.

LECANIUM nigrum.

HeMichIONASPIS minor.

GUETTARDA (Rubiaceae).

HowaRdia biclavis.

GUTIERREZIA (Compositae).

Palaeococcus townsendi,
ORTHEZIA nigrocincta.

TACHARDia glomerella.

Eriococcus tinsleyi-cryptus.

Pseudococcus gutierreziae, neomexicanus.

Aspidiotus gutierreziae.

GYMNOSPORIA (Celastraceae).

LECANIUM gymnospori.

Aspidiotus gymnosporiae, lauretorum.

GYNANDROPSIS (Capparidaceae).

CHIOnaspis gynandropsidis.

HABROTHAMNUS (Solanaceae).

Pseudococcus citri.

HAKEA (Proteaceae).

Palaeococcus australis, rosae.

Asterolecanium hakeae.

Eriococcus hakeae.

LECANIÚm depressum.

Pulvinaria maskelli-viminariae.

Austrolichtensia hakearum.

Chionaspis eugeniae.

Aspidiotus hakeae, comperei, acaciae-propinqua.

Aonidia rotunda.

Gymnaspis perpusilla.

Parlatoria petrophilae.

LEPIDOSAPHES defecta-tincta.

HALOXYLON (Chenopodiaceae).

Pulvinaria orientalis.

HAMELIA (Rubiaceae).

Orthezla insignis.

HARPULLIA (Sapindaceae).

TACHARdia albizziae.

HAROGANA (Guttiferae).

Stictococcus gowdeyi.

INGLISIA conchiformis.

HEBERDENIA (Myrsinaceae).

Aspidiotus lauretorum.

HEDERA (Araliaceae) 'Ivy.'

ICERYA purchasi.

ORthezia urticae.

Asterolecanium hederae, fimbriatum.

Pseudococcus citri.

Phenacoccus hederae, aceris.

LeCaniUm hesperidum, maculatum.

Filippia oleae.

Lichtensia viburni. 
HEDERA (Araliaceae)-cont.

Diaspis bromeliae.

Aspidiotus cydoniae-crawi, hederae, lauretorum, camelliae, ficus, dictyospermi, britannicus.

HEDYCARPUS (Euphorbiaceae).

LEUCASPIS stricta.

HEDYCARYA (Monimiaceae).

Ctenochiton viridis.

HEDYOTIS (Rubiaceae).

ChIONASPIS hedyotidis, galliformens.

HELIANTHEMUM (Cistaceae).

Newsteadia floccosa.

ASTEROLECANIUM fimbriatum.

LECANIODIASPIS sardoa.

Cerococcus eremobius.

LEPIDOSAPHES ulmi.

HELIANTHUS (Compositae).

Phenacoccus helianthi.

Aspidiotus helianthi.

HELICHRYSUM (Compositae).

Cerococcus bryoides.

ErIococcus sordidus.

LECANIUM oleae.

Aspidiotus niger.

HELICONIA (Musaceae).

Diaspis boisduvallii.

HeMichionaspis aspidistrae.

HELICTERES (Sterculiaceae).

Cerococcus indicus.

ERIococcus paradoxus-indicus.

HELIOTROPIUM (Boragineae).

DIASPIS pentagona.

Chionaspis major.

HEMICYCLEA (Euphorbiaceae).

TACHARDIA albizziae.

LECANIUM expansum.

Aonidia echinata.

HERACLEUM (Compositae).

OrthezIa urticae.

HERNIARIA (Caryophyllaceae).

Margarodes polonicus.

HETERODENDRON (Sapindaceae).

TACHaRdia melaleucae.

Aspidiotus hederae.

HEVEA (Euphorbiaceae).

Asterolecanium pustulans-seychellarum.

Pseunococcus virgatus.

LeCANIUM nigrum.
Vinsonia stellifera.

Chinoaspis dilatata.

HемптенIONASPIS aspidistrae.

Aspidiotus cyanophylli, destructor, transparens, ficus, personatus.

Parlatoria proteus.

LEPIDOSAPHES rubrovittatus.

HIBBERTIA (Dilleniaceae).

Pseudococcus hibbertiae.

HIBISCUS (Malvaceae).

Conchaspis angraeci-hibisci.

Asterolecanium pustulans.

Cerococcus hibisci.

Pseudococcus bromeliae, cocotis, filamentosus.

LECANIUM hesperidum, depressum, nigrum.

Pulvinaria cestri, citricola.

Ericerus pela.

Ceroplastes ceriferus, dugesii, ficus.

INGLISIA malvacearum.

Stictococcus diversiseta.

DiAsPIs bromeliae, pentagona.

Howardia biclavis.

HeMichIONASPIS minor, mussaendae, aspidistrae.

Aspidiotus longispinus, perniciosus, cydoniae.

Parlatoria chinensis.

HIERACEUM (Compositae).

Margarodes polonicus.

OrTHEZIA urticae.

ASTERoleCANIUM fimbriatum.

Ceroputo pilosellae.

HIPPEASTRUM (Amaryllidaceae).

Pseudococcus citri.

HIPPOCREPIS (Leguminosae).

AsterolecaniUm fimbriatum.

HIPPOPHAES (Elaeagnaceae).

Phenacoccus aceris.

Chionaspis salicis.

LEPIDOSAPHES ulmi.

HIPTAGE (Malpighiaceae).

LECANIUM tessellatum, viride.

HOHERIA (Malvaceae).

ERIococcus hoheriae.

Chionaspis dysoxyli.

LEUCASPIS stricti.

HOLACANTHA (Simarubaceae).

Diaspis toumeyi. 
HOMALOMENA (Araceae).

Pinnaspis buxi.

HOMOGYNE (Compositae).

Orthezia cataphracta.

HORDEUM (Gramineae).

Pseudococcus hordei.

HOSTA (Liliaceae).

Hemichionaspis aspidistrae.

HOWEA (Palmaceae).

Lecanium tessellatum.

Fiorinia kewensis.

HOYA (Asclepiadaceae).

Pseudococcus hoyae.

HUMBOLDTIA (Leguminosae).

LACHNODIUS humboldtiae.

HUMULUS (Urticaceae) 'Hop.'

Orthezia urticae.

Phenacoccus aceris.

HURA (Euphorbiaceae).

LECANIUM oleae, hurae.

Ceroplastes cirripediformis.

HYALIS (Compositae).

Pseudococcus mendozinus.

HYDRANGEA (Saxifragaceae).

LECANIUM persicae, magnoliarumhortensiae.

HYGROPHILA (Acanthaceae).

Pseudococcus viridis.

Lecanium nigrum, oleae.

Pulvinaria obseura.

HYMENAEA (Leguminosae).

Lichtensia zapotlana-townsendi.

Howardia biclavis.

HYMENANTHERA (Violaceae).

Pulvinaria maskelli-novemarticulata.

Ctenochton hymenantherae.

LePIDOSAPHES hymenantherae.

HYMENOCLEA (Compositae).

Orthezia sonorensis.

Pseudococcus hymenocleae.

HYPERICUM (Guttiferae).

Asterolecanium fimbriatum.

LECANIUM pulchrum.

Aspidiotus privignus, camelliae, labiatarum.

HYPHAENA (Palmaceae).

Chionaspis pseudonivea.

Aspidiotus fissidens-constricta.

HYPOCALYMMA (Myrtaceae).
TACHARDia convexa.

Sphaerococcus pulchellus.

HYPTIS (Labiatae).

DiAsPIs pentagona.

HYSSOPUS (Labiatae).

Aspidiotus rossi.

IDESIA (Flacourtiaceae).

Pulvinaria idesiae.

ILEX (Aquifoliaceae) 'Holly.'

Palaeococcus pulcher.

LECANIUM hesperidum, notatum, nigrofasciatum, oleae.

Ceronema japonica.

Ceroplastes floridensis, grandis, rubens, rusci.

Diaspis penzigi.

Pinnaspis javanica.

Aspidiotus britannicus, hederae, camelliae, ficus, perseae, lauretorum, paeoniae, dictyospermi.

Parlatoria theae-viridis.

ILLIGERA (Combretaceae).

Aspidiotus replicatus.

IMPATIENS (Geraniaceae).

Ceroplastes floridensis.

INDIGOFERA (Leguminosae).

Hemichionaspis ehionaspiformis, minor.

INGA (Leguminosae).

ICERYA montserratensis.

TaCHaRdia ingae.

Asterolecanium pustulans.

Ceroplastes confluens.

IPOMOEA (Convolvulaceae).

ORTHEzIA insignis.

Pseudococcus citri.

Lecanium hesperidum, batatae.

Pulvinaria urbicola.

Ceroplastodes cajani.

Ceroplastes cirripediformis.

Hemichionaspis minor.

Aspmiotus cyanophylli, excisus.

IRIS (Iridaceae).

Aspidiotus dictyospermi.

IXORA (Rubiaceae).

LECANIUM mangiferae, hemisphaericum, maritimum, hesperidum, viride.

Pulvinaria ixorae, 
IXORA (Rubiaceae)-cont.

Aspidiotus trilobitiformis, articulatus.

LEPIDOSAPHES ixorae.

IsCHNASPIS filiformis.

JABOTICABA (Myrtaceae).

Aspidiotus trilobitiformis.

JASMINUM (Oleaceae).

ICERYA purchasi.

AsterolecaniUm pustulans.

Phenacoccus ornatus.

Lecanium hesperidum, mangiferae, acuminatum.

Diaspis pentagona.

Chionaspis euonymi.

HowARDIA biclavis.

Aspidiotus lataniae, forbesi, personatus, dictyospermi, aurantii, rossi, hederae, trilobitiformis, britannicus.

Parlatoria blanchardi.

IsCHNASPIS filiformis.

JATROPHA (Euphorbiaceae).

LLAVEIA axin.

Pseddococcus virgatus.

LECANIUM nigrum.

HeMmLECANIUM imbricans.

HeMtCHIONASPIS minor.

JOSSINIA (Myrtaceae).

Pulvinaria grabhami.

JUGLANS (Juglandaceae) 'Walnut.'

Pseudococcus bakeri, citrophilus.

Lecanium cockerelli, juglandifex, juglandis, oleae, coryli.

Pulvinaria betulae.

Diaspis pentagona.

Chionaspis furfura, ortholobis.

EPIDIASPIS lepèri.

Aspidiotus aesculi-solus, forbesi, juglandis, juglans-regiae, perniciosus, ulmi.

LePIDOSAPHES juglandis, ulmi, multipora, conchiformis.
JUNIPERUS (Coniferae).

ErIococcus gillettei.

Pseudococcus juniperi, vovae.

Diaspis juniperi, carueli, atlantica, visci.

Chionaspis striata.

AONIDIA juniperi.

Cryptoparlatoria leucaspis.

Aspidiotus maderensis.

Cryptaspidiotus mediterraneus.

Fiorinta juniperi.

LEPIDOSAPHES juniperi.

JUSTICIA (Acanthaceae).

ORTHEZIA insignis.

LECANIUM hemisphaericum, nigrum, viride.

Chionaspis longispina.

KENNEDYA (Leguminosae).

ICERYA purchasi.

Aspidiotus kennedyae.

KENTIA (Palmaceae).

Pseudococcus pseudonipae, nipae.

Diaspis boisduvallii.

Fiorinia pellucida.

Aspidiotus hederae, dictyospermi, ficus.

Parlatoria proteus.

KHAYA (Meliaceae).

DiAspis senegalensis.

KIBARA (Monimiaceae).

Myxolecanium kibarae.

KIGELIA (Bignoniaceae).

Aspidiotus lataniae.

KNIGHTIA (Proteaceae).

ERIococcus multispinus.

KOELREUTERIA (Sapindaceae).

Pulvinaria horii.

KRAUNHIA (Leguminosae).

LECANIUM corni, persicae.

KUNZEA (Myrtaceae).

ERIococcus leptospermi, araucariaminor.

KYDIA (Malvaceae).

TACHARDIA lacca.

CAMbridge: PRinted By J. B. PEACE, M.A., at the University press. 


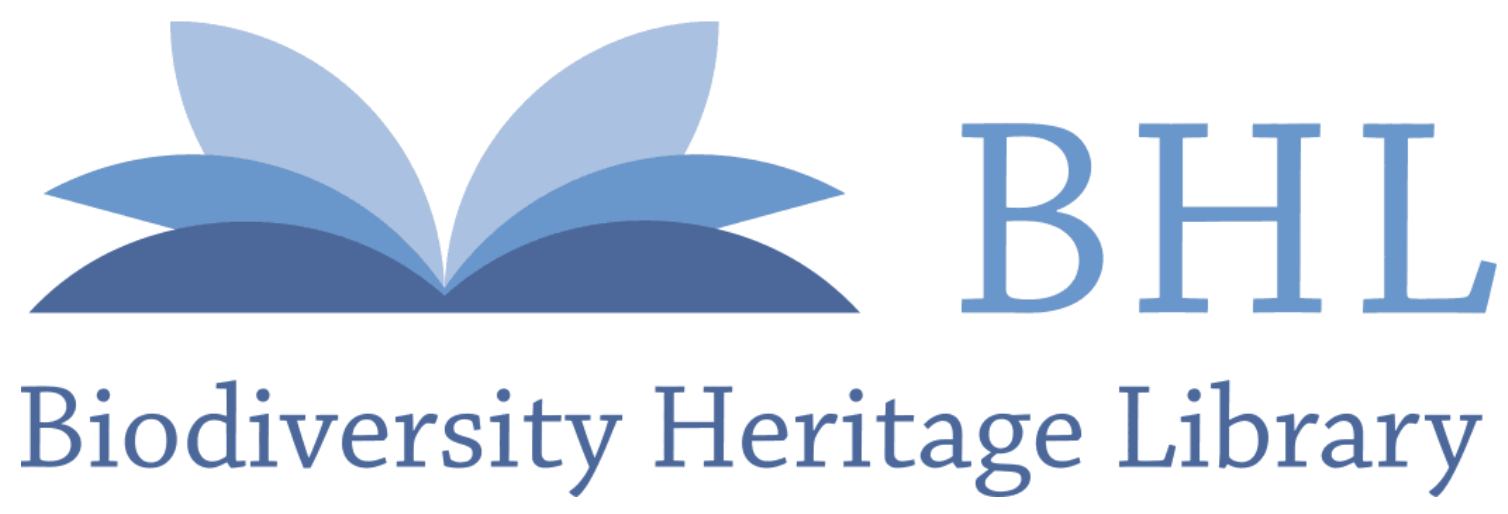

Green, Edward Ernest. 1918. "A list of Coccidae affecting various genera of plants." Annals of applied biology 4, 228-239.

View This Item Online: https://www.biodiversitylibrary.org/item/33699

Permalink: https://www.biodiversitylibrary.org/partpdf/31855

\section{Holding Institution}

MBLWHOI Library

\section{Sponsored by}

MBLWHOI Library

\section{Copyright \& Reuse}

Copyright Status: NOT_IN_COPYRIGHT

This document was created from content at the Biodiversity Heritage Library, the world's largest open access digital library for biodiversity literature and archives. Visit BHL at https://www.biodiversitylibrary.org. 